\title{
Effect of transcatheter arterial chemoembolization prior to surgical resection for hepatocellular carcinoma
}

\author{
HIROKI NISHIKAWA ${ }^{1}$, AKIRA ARIMOTO ${ }^{2}$, TOMOKO WAKASA ${ }^{3}$, \\ RYUICHI KITA $^{1}$, TORU KIMURA ${ }^{1}$ and YUKIO OSAKI ${ }^{1}$ \\ Departments of ${ }^{1}$ Gastroenterology and Hepatology, ${ }^{2}$ Surgery and ${ }^{3}$ Pathology, \\ Osaka Red Cross Hospital, Tennoji-ku, Osaka 543-0027, Japan
}

Received September 11, 2012; Accepted October 22, 2012

DOI: 10.3892/ijo.2012.1711

\begin{abstract}
The aim of the present study was to evaluate the influence of preoperative transcatheter arterial chemoembolization (TACE) on survival after surgical resection (SR) for hepatocellular carcinoma (HCC). Two hundred and thirty-five HCC patients who underwent SR with curative intent were analyzed. Overall survival (OS), recurrence-free survival (RFS) and complication rates were compared between the TACE $(n=110)$ and control groups $(n=125)$. Moreover, TACE subjects were classified into TACE responders $(n=85)$ and TACE non-responders $(n=25)$, according to the therapeutic efficacy of pretreatment TACE, and the factors contributing to OS and RFS after SR were analyzed using univariate and multivariate analyses. The 1-, 3- and 5-year OS rates were 87.4, 76.0 and $62.5 \%$, respectively, in the TACE group and 94.9, 79.0 and $57.8 \%$, respectively, in the control group $(\mathrm{P}=0.674)$. The corresponding RFS rates at 1,3 and 5 years were $73.3,48.9$ and $33.2 \%$, respectively, in the TACE group and 73.3, 29.4 and $16.3 \%$, respectively, in the control group $(\mathrm{P}=0.062)$. No TACErelated serious adverse events (SAEs) were observed. There were no significant differences between the groups in terms of surgery-related SAEs $(\mathrm{P}=0.714)$, operation time $(\mathrm{P}=0.881)$, blood loss during surgery $(\mathrm{P}=0.334)$ and hospitalization period $(\mathrm{P}=0.447)$. Multivariate analyses identified TACE responder, TACE non-responder, total bilirubin $\geq 1 \mathrm{mg} / \mathrm{dl}$, serum albumin $\geq 4 \mathrm{~g} / \mathrm{dl}$, pretreatment $\alpha$-fetoprotein (AFP) level $\geq 100 \mathrm{ng} / \mathrm{ml}$ and microscopic vascular invasion as significant prognostic factors linked to OS. TACE non-responder, tumor number (multiple) and pretreatment AFP level $\geq 100 \mathrm{ng} / \mathrm{ml}$ were significant adverse prognostic factors linked to RFS. In conclusion, TACE is a safe procedure in patients with HCC, and the efficacy of TACE prior to surgery may be associated with clinical outcomes after SR.
\end{abstract}

Correspondence to: Dr Hiroki Nishikawa, Department of Gastroenterology and Hepatology, Osaka Red Cross Hospital, 5-30 Fudegasaki-cho, Tennoji-ku, Osaka 543-0027, Japan

E-mail: h-nishikawa@osaka-med.jrc.or.jp

Key words: hepatocellular carcinoma, pretreatment transcatheter arterial chemoembolization, treatment efficacy, surgical resection, prognosis

\section{Introduction}

Hepatocellular carcinoma (HCC) is a major health problem worldwide, with an estimated incidence ranging between 500,000 and 1,000,000 new cases annually. It is the fifth most common cancer in the world and the third most common cause of cancer-related death $(1,2)$. The prognosis of HCC is generally poor because of the high recurrence rate $(1,3,4)$. Surgical resection (SR) remains the best curative treatment, but is only suitable in $9-27 \%$ of patients. The presence of significant background liver cirrhosis often precludes hepatic resection in patients with HCC. Recurrence in the liver remnant is also common in patients who have undergone radical hepatic resection (5).

Transcatheter arterial chemoembolization (TACE) is one of the available locoregional therapies for HCC. It involves injection of an embolizing agent into the hepatic artery to deprive the tumor of its major nutrient source via embolization of the nutrient artery, resulting in ischemic necrosis of the tumor (6-8). According to the current guidelines, TACE is generally performed in intermediate-stage HCC patients (9). However, TACE has also been performed as preoperative adjuvant chemotherapy in resectable HCC patients with the aim of improving survival $(3,10,11)$.

The purpose of preoperative TACE is to reduce tumor volume, induce tumor necrosis and prevent cancer cell dissemination during the surgical procedure $(3,10,11)$. To the best of our knowledge, four randomized controlled trials have assessed the efficacy of preoperative TACE in terms of survival $(3,10,12,13)$. However, the results of these trials are difficult to compare because of differences in baseline clinical characteristics such as tumor size, cause of liver disease and chemotherapeutic agents used when performing TACE. Hence, the postoperative survival benefits of preoperative TACE for HCC remain a matter of debate.

The aim of the present study was to evaluate the influence of preoperative TACE on survival after SR for HCC.

\section{Patients and methods}

Patients. SR was performed in 342 treatment-naïve HCC patients at the Department of Surgery, Osaka Red Cross Hospital, Japan, between January 2004 and June 2012. Of these, we excluded patients operated on without curative intent 
$(n=41)$, with surgery-related death $(n=3)$, with TACE alone before surgery $(\mathrm{n}=10)$, and with transcatheter arterial lipiodol chemolization alone before surgery $(n=53)$. We defined curative surgery as the resection of all tumors detectable using imaging modalities. A total of 235 HCC patients who underwent SR were therefore analyzed in the present study (Fig. 1), including 110 patients who underwent TACE before surgery (TACE group) and 125 patients who did not (control group). All patients in the TACE group received one session of TACE, and patients in the control group received angiography alone. The decision to perform TACE prior to surgery was made mainly by the attending physician. Written informed consent was obtained from all patients prior to TACE and surgery, and the study protocol complied with all of the provisions of the Declaration of Helsinki. The present study comprised a retrospective analysis of patient records, and all treatments were conducted in an open-label manner.

HCC diagnosis and stage. HCC was diagnosed using abdominal ultrasound and dynamic computed tomography (CT) scans (hyperattenuation during the arterial phase in all or some part of the tumor and hypoattenuation in the portal-venous phase) and/or magnetic resonance imaging (MRI), based mainly on the recommendations of the American Association for the Study of Liver Diseases (9). Arterial and portal phase dynamic CT images were obtained at $\sim 30$ and $120 \mathrm{sec}$, respectively, after the injection of the contrast material. Abdominal angiography combined with CT assistance was performed on all patients before SR, in line with the recommendations of Yamasaki et al, who reported that this technique was useful for detecting small satellite nodules (14). HCC stage was determined using the Liver Cancer Study Group of Japan (LCSGJ) staging system (15). HCC was confirmed pathologically in resected specimens at surgery, except for cases with complete necrosis.

TACE procedure. TACE was performed in accordance with Japanese guidelines (16), and consisted of catheterization via the femoral artery with super-selective cannulation to the hepatic artery feeding the target HCC. An emulsion containing Farmorubicin (epirubicin hydrochloride; Pfizer), mitomycin C (Kyowa Hakko Kirin Co. Ltd., Tokyo, Japan) and lipiodol (iodine addition products of ethyl esters of fatty acids obtained from poppy seed oil; Mitsui, Japan) was infused via the feeding artery according to tumor size, tumor number and liver function. Embolization was then achieved by slow injection of gelatin (Spongel; Yamanouchi, Japan) to prevent reflux into untreated segments. The injection sites were segmental or subsegmental in all TACE group patients. The mean doses of epirubicin, mitomycin and lipiodol in the TACE group were $38.0 \pm 12.5 \mathrm{mg}$ (range $10-70 \mathrm{mg}$ ), $8.9 \pm 3.1 \mathrm{mg}$ (range $2-14 \mathrm{mg}$ ) and $5.2 \pm 2.7 \mathrm{ml}$ (range $1-15 \mathrm{ml}$ ), respectively.

Treatment efficacy of TACE. The treatment efficacy of TACE was classified into four grades according to the Response Evaluation Criteria in Cancer of the Liver proposed by the LCSGJ (17) and based on CT scans performed within 30 days after TACE: TE4, tumor-necrotizing effect of 100\%; TE3, tumor-necrotizing effect of 50 to $<100 \%$; TE2, effects other than TE3 and TE1; TE1, tumor enlarged by $>25 \%$ regardless of the necrotizing effect. In the present study, we defined

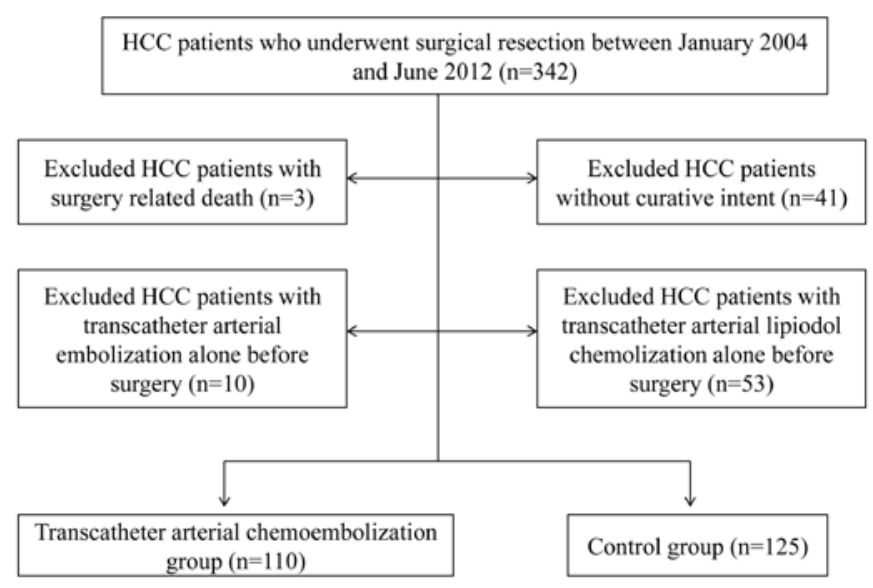

Figure 1. Study profile.

TACE responders as TE4 or TE3 and TACE non-responders as TE2 or TE1.

SR procedure. Conventional open hepatectomy was performed in 179 patients (76.2\%) and laparoscopic hepatectomy was performed in 56 patients (23.8\%). All procedures were performed by one of four surgeons who had at least 10 years of experience of SR.

Conventional open hepatectomy was carried out under general anesthesia using a right subcostal incision with a midline extension. We performed anatomic partial hepatectomy with a resection margin of at least $1 \mathrm{~cm}$ over the tumor, based on intraoperative ultrasonography (IOUS) guidance. IOUS was performed routinely to estimate the location, size, number and feeding vessels of the tumor, as well as to provide a clear vascular map of the liver anatomy. The Cavitron Ultrasonic Aspiration system (CUSA; Valley Lab Corp., Boulder, CO, USA) was used to dissect the liver tissue. Hemostasis was achieved by dipolar electric coagulation and suturing. The Pringle maneuver was usually used in cases with cirrhotic liver, with a clamp/unclamp time of $15 / 5$ min policy.

Laparoscopic hepatectomy was performed using the fourtrocar technique. The first trocar was placed by a small incision below the umbilicus for pneumoperitoneum creation. The tumor extent and its relationship with the vascular anatomy and other tumors in the liver were explored using IOUS. The line of the intended liver parenchymal transection was marked on the surface of the liver using diathermy. Ultrasonic dissection was performed using an ultrasonic surgical system. The resected liver was maneuvered into a plastic bag (18). Patients were discharged when their liver function returned to normal and any adverse events were resolved.

Follow-up. Follow-up consisted of periodic blood tests and monitoring of tumor markers, including $\alpha$-fetoprotein (AFP) and des- $\gamma$-carboxy prothrombin (DCP), measured using a chemiluminescent enzyme immunoassay (Lumipulse PIVKAII Eisai, Eisai, Tokyo, Japan). Dynamic CT scans and/ or MRI were obtained every 3-4 months after SR. Chest CT, whole abdominal $\mathrm{CT}$ and bone scintigraphy were performed when extrahepatic HCC recurrences were suspected. 
Table I. Baseline characteristics between the TACE group and the control group.

\begin{tabular}{lccc}
\hline & TACE group $(\mathrm{n}=110)$ & Control group $(\mathrm{n}=125)$ & P-value \\
\hline Gender (male/female) & $86 / 24$ & $93 / 32$ & $0.541^{\mathrm{a}}$ \\
Age (years) & $67.7 \pm 10.2$ & $68.1 \pm 10.5$ & $0.739^{\mathrm{b}}$ \\
HCC stage (I/II/III/IV) & $5 / 57 / 38 / 10$ & $11 / 82 / 25 / 7$ & $0.029^{\mathrm{a}}$ \\
Etiology (HBV/HCV/nBnC) & $15 / 63 / 32$ & $15 / 74 / 36$ & $0.922^{\mathrm{a}}$ \\
Child-Pugh classification (A/B) & $108 / 2$ & $120 / 5$ & $0.453^{\mathrm{a}}$ \\
Tumor number (single/multiple) & $65 / 45$ & $94 / 31$ & $0.012^{\mathrm{a}}$ \\
Maximum tumor size (cm) & $5.0 \pm 3.2$ & $4.5 \pm 2.2$ & $0.138^{\mathrm{b}}$ \\
AST (IU/l) & $59.5 \pm 40.2$ & $55.6 \pm 35.4$ \\
ALT (IU/l) & $53.7 \pm 45.1$ & $49.6 \pm 37.3$ & $3.94 \pm 0.52$ \\
Serum albumin (g/dl) & $3.94 \pm 0.52$ & $0.83 \pm 0.43$ \\
Total bilirubin (mg/dl) & $0.78 \pm 0.39$ & $91.2 \pm 14.8$ \\
Prothrombin time (\%) & $89.3 \pm 13.8$ & $14.8 \pm 6.8$ \\
Platelets (x10 $\left./ \mathrm{mm}^{3}\right)$ & $15.0 \pm 7.8$ & $14.6 \pm 10.4$ \\
ICGR 15 (\%) & $12.7 \pm 9.3$ & $1,064.5 \pm 4,370.0$ \\
AFP (ng/ml) & $2,841.0 \pm 15,411.0$ & $2,017.5 \pm 5,625.6$ & $0.964^{\mathrm{b}}$ \\
DCP (mAU/ml) & $6,901.1 \pm 23,091.8$ & $0.338^{\mathrm{b}}$ \\
\hline
\end{tabular}

TACE, transcatheter arterial chemoembolization; HCC, hepatocellular carcinoma; HBV, hepatitis B virus; HCV, hepatitis C virus; AST, aspartate aminotransferase; ALT, alanine aminotransferase; ICGR 15, indocyanine green retention at 15 min; AFP, $\alpha$-fetoprotein; DCP, des- $\gamma$ carboxy prothrombin. ${ }^{\mathrm{a} F i s h e r ' s ~ e x a c t ~ t e s t ; ~}{ }^{\mathrm{b}}$ unpaired t-test.

Statistical analysis. The primary endpoints were overall survival (OS) and recurrence-free survival (RFS), and the secondary endpoints were procedure-related complications. Data were analyzed using univariate and multivariate analyses. Continuous variables were compared using unpaired t-tests and categorical variables were compared using Fisher's exact tests. Time to recurrence was defined as the interval between surgery and first confirmed recurrence. For analysis of RFS, follow-up ended at the time of first recurrence; other patients were censored at their last follow-up visit or the time of death from any cause without recurrence. For analysis of OS, followup ended at the time of death from any cause, and the remaining patients were censored at the last follow-up visit. The cumulative OS and RFS rates were calculated using the Kaplan-Meier method and tested using log-rank tests. The Cox proportional hazards model was used for multivariate analysis of factors that were considered significant in univariate analysis. These statistical methods were used to estimate the interval from surgery. Data were analyzed using SPSS software, version 9.0 (SPSS Inc., Chicago, IL, USA) for Microsoft Windows. Data are expressed as means \pm standard deviation. Values of $\mathrm{P}<0.05$ were considered to be statistically significant.

\section{Results}

Patients. Baseline characteristics of the TACE and control groups are shown in Table I. The mean observation periods were $2.8 \pm 1.8$ years in the TACE group and $2.9 \pm 2.1$ years in the control group. There were significant differences between the two groups in terms of HCC stage, tumor number and pretreatment DCP-value, indicating that patients in the TACE group had more advanced tumor characteristics. Anatomical resection was performed in 54 patients in the TACE group, and non-anatomical resection in 56 patients, compared with 57 and 68 patients in the control group $(\mathrm{P}=0.603)$.

Histological findings. The histological findings in the TACE and control groups are shown in Table II. Complete necrosis occurred in 21 patients (19.1\%) in the TACE group. Microscopic vascular invasion was found in 32 patients $(29.1 \%)$ in the TACE group and 47 patients $(37.6 \%)$ in the control group.

Cumulative OS and RFS in the TACE and control groups. The 1-, 3- and 5-year OS rates in the two groups were 87.4, 76.0 and $62.5 \%$, respectively, in the TACE group and 94.9, 79.0 and $57.8 \%$, respectively, in the control group (Fig. 2). There was no significant difference in OS between the two groups $(\mathrm{P}=0.674)$. The corresponding RFS rates at 1, 3 and 5 years were $73.3,48.9$ and $33.2 \%$, respectively, in the TACE group and $73.3,29.4$ and $16.2 \%$, respectively, in the control group (Fig. 3). RFS was higher in the TACE group, but the difference was not significant $(\mathrm{P}=0.062)$.

OS and RFS in TACE responders and non-responders. In terms of the efficacy of TACE, 21 patients were classified as TE4, 64 as TE3, 25 as TE2 and 0 as TE1. The TACE group was further categorized into TACE responders (TE4 and TE3; $\mathrm{n}=85$ ) and TACE non-responders (TE2 and TE1; $\mathrm{n}=25$ ), and OS and RFS were compared between the TACE responders, TACE non-responders, and the control group. There were significant differences in OS between the three groups (TACE responders vs. controls, $\mathrm{P}=0.381$; controls vs. TACE non-responders, 
Table II. Type of surgery, outcome of surgery and histological findings between the TACE group and the control group.

\begin{tabular}{lcc}
\hline Variables & TACE group $(\mathrm{n}=110)$ & Control group (n=125) \\
\hline Hepatectomy & & $57 / 68$ \\
Anatomical/non-anatomical & $54 / 56$ & $269.1 \pm 87.8$ \\
Operation time (min) & $259.5 \pm 74.5$ & $0.603^{\mathrm{a}}$ \\
Blood loss during surgery (ml) & $764.1 \pm 713.5$ & $16.6 \pm 10.4$ \\
Hospitalization days & $17.9 \pm 15.8$ & $0.334^{\mathrm{b}}$ \\
HCC histology & & 14 \\
Well & 7 & 73 \\
Moderate & 47 & 38 \\
Poorly & 35 & $0.447^{\mathrm{b}}$ \\
Complete necrosis & 21 & 95 \\
Fibrous capsule (yes) & 90 & 81 \\
Capsular invasion (yes) & 52 & 47 \\
Microscopic vascular invasion (yes) & 32 & 40 \\
Microscopic surgical margin (yes) & 24 & 38.8 \\
\hline
\end{tabular}

HCC, hepatocellular carcinoma; TACE, transcatheter arterial chemoembolization. ${ }^{\mathrm{a} F i s h e r ' s ~ e x a c t ~ t e s t ; ~}{ }^{\mathrm{b}}$ unpaired t-test.

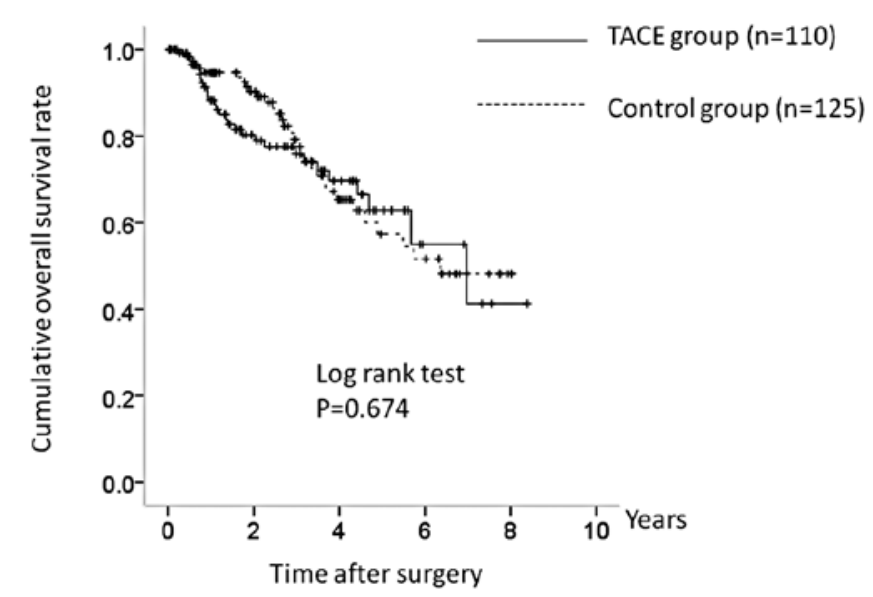

Figure 2. Cumulative overall survival (OS) rates in the transcatheter arterial chemoembolization (TACE) group $(n=110)$ and the control group $(n=125)$. The 1-, 3- and 5-year OS rates were 87.4, 76.0 and $62.5 \%$, respectively, in the TACE group and $94.9,79.0$ and $57.8 \%$, respectively, in the control group. There was no significant difference between the two groups in terms of OS $(\mathrm{P}=0.674)$.

$\mathrm{P}<0.001 ;$ TACE responders vs. TACE non-responders, $\mathrm{P}<0.001$; overall significance, $\mathrm{P}<0.001$ ) (Fig. 4). There were also significant differences in RFS between the three groups (TACE responders vs. controls, $\mathrm{P}=0.006$; controls vs. TACE non-responders, $\mathrm{P}=0.190$; TACE responders vs. TACE nonresponders, $\mathrm{P}=0.004$; overall significance, $\mathrm{P}=0.004)($ Fig. 5).

Factors contributing to $O S$ after $S R$. Univariate analysis identified pretreatment therapy $(\mathrm{P}<0.001)$, HCC stage $(\mathrm{P}=0.012)$, maximum tumor size $\geq 4 \mathrm{~cm}(\mathrm{P}=0.003)$, tumor number $(\mathrm{P}=0.005)$, total bilirubin $\geq 1 \mathrm{mg} / \mathrm{dl}(\mathrm{P}=0.003)$, serum albumin $\geq 4.0 \mathrm{~g} / \mathrm{dl}(\mathrm{P}=0.013)$, AFP $\geq 100 \mathrm{ng} / \mathrm{ml}(\mathrm{P}<0.001)$, $\mathrm{DCP} \geq 100 \mathrm{mAU} / \mathrm{ml}(\mathrm{P}=0.020)$ and microscopic vascular invasion $(\mathrm{P}=0.002)$ as significant factors contributing to $\mathrm{OS}$

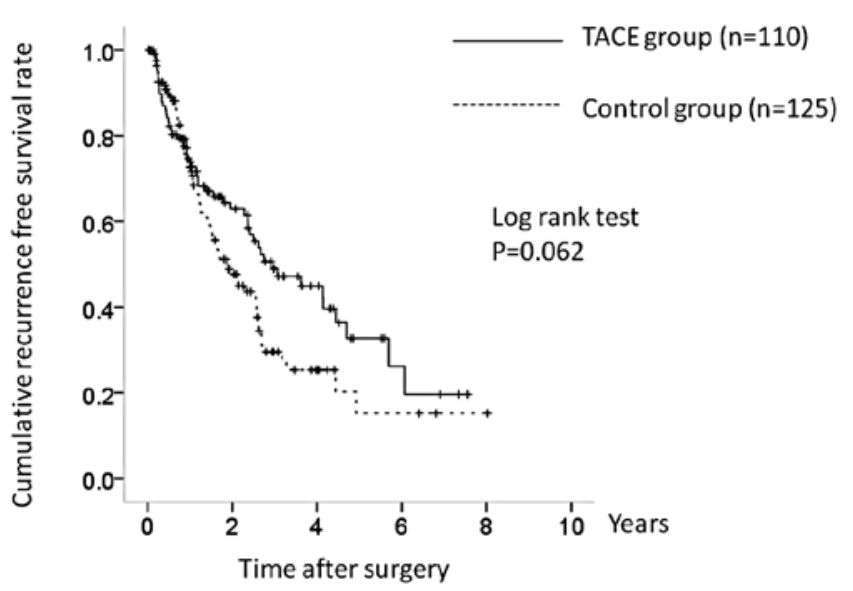

Figure 3. Cumulative recurrence-free survival (RFS) rate in the transcatheter arterial chemoembolization (TACE) group $(\mathrm{n}=110)$ and the control group $(\mathrm{n}=125)$. The 1-, 3- and 5-year RFS rates were 73.3, 48.9 and $33.2 \%$, respectively, in the TACE group and 73.3, 29.4 and 16.2\%, respectively, in the control group. The RFS rate was higher in the TACE group compared with the control group, but the difference was not significant $(\mathrm{P}=0.062)$.

after SR (Table III). Multivariate analysis of the nine factors found to be significant in univariate analysis further identified pretreatment therapy (TACE responder, $\mathrm{P}=0.018$ ), pretreatment therapy (TACE non-responder, $\mathrm{P}=0.019$ ), total bilirubin $\geq 1 \mathrm{mg} / \mathrm{dl}(\mathrm{P}=0.003)$, serum albumin $\geq 4.0 \mathrm{~g} / \mathrm{dl}$ ( $\mathrm{P}=0.001)$, AFP $\geq 100 \mathrm{ng} / \mathrm{ml}(\mathrm{P}=0.011)$ and microscopic vascular invasion $(\mathrm{P}=0.021)$ as significant contributors to $\mathrm{OS}$. The hazard ratios (HRs) and 95\% confidence intervals (CIs) for these factors are detailed in Table IV.

Factors contributing to RFS after SR. Univariate analysis identified pretreatment therapy $(\mathrm{P}=0.004)$, HCC stage $(\mathrm{P}<0.001)$, maximum tumor size $\geq 4 \mathrm{~cm}(\mathrm{P}=0.049)$, tumor 
Table III. Univariate analyses contributing to OS and RFS after surgical resection ( $\mathrm{n}=235)$.

\begin{tabular}{|c|c|c|c|}
\hline & & OS & RFS \\
\hline Variables & $\mathrm{n}$ & P-value ${ }^{a}$ & P-value ${ }^{a}$ \\
\hline Age $\geq 70$ (yes/no) & $119 / 116$ & 0.229 & 0.794 \\
\hline Gender (male/female) & $179 / 56$ & 0.504 & 0.848 \\
\hline \multicolumn{4}{|l|}{ Pretreatment therapy } \\
\hline TACE-R/TACE-NR/controls & $85 / 25 / 125$ & $<0.001$ & 0.004 \\
\hline \multicolumn{4}{|l|}{ Cause of liver disease } \\
\hline Hepatitis B/hepatitis C/non-Bnon-C & $30 / 137 / 68$ & 0.713 & 0.935 \\
\hline HCC stage (I, II/III, IV) & $155 / 80$ & 0.012 & $<0.001$ \\
\hline Maximum tumor size $\geq 4 \mathrm{~cm}$ (yes $/ \mathrm{no})$ & $115 / 120$ & 0.003 & 0.049 \\
\hline Tumor number (single/multiple) & $159 / 76$ & 0.005 & $<0.001$ \\
\hline ICGR $15 \geq 12 \%$ & $113 / 122$ & 0.289 & 0.319 \\
\hline Total bilirubin $\geq 1.0 \mathrm{mg} / \mathrm{dl}$ (yes $/ \mathrm{no}$ ) & $62 / 173$ & 0.003 & 0.041 \\
\hline Serum albumin $\geq 4.0 \mathrm{~g} / \mathrm{dl}$ (yes/no) & $125 / 110$ & 0.013 & 0.231 \\
\hline $\mathrm{AST} \geq 50 \mathrm{IU} / 1(\mathrm{yes} / \mathrm{no})$ & $106 / 129$ & 0.353 & 0.005 \\
\hline ALT $\geq 50$ IU/l (yes/no) & $90 / 145$ & 0.263 & 0.012 \\
\hline Platelets $\geq 10 \times 10^{4} / \mathrm{mm}^{3}$ (yes $\left./ \mathrm{no}\right)$ & $172 / 63$ & 0.502 & 0.549 \\
\hline Prothrombin time $\geq 80 \%$ (yes/no) & $177 / 58$ & 0.112 & 0.144 \\
\hline $\mathrm{AFP} \geq 100 \mathrm{ng} / \mathrm{ml}$ (yes/no) & $70 / 165$ & $<0.001$ & 0.036 \\
\hline $\mathrm{DCP} \geq 100 \mathrm{mAU} / \mathrm{ml}$ (yes/no) & $151 / 84$ & 0.020 & 0.180 \\
\hline Microscopic capsule (yes/no) & $185 / 50$ & 0.696 & 0.171 \\
\hline Microscopic capsule invasion (yes/no) & $133 / 102$ & 0.147 & 0.520 \\
\hline Microscopic vascular invasion (yes/no) & $80 / 155$ & 0.002 & 0.021 \\
\hline Microscopic surgical margin (yes/no) & $64 / 171$ & 0.818 & 0.951 \\
\hline
\end{tabular}

OS, overall survival; RFS, recurrence-free survival; TACE-R, TACE responders; TACE-NR, TACE non-responders; HCC, hepatocellular carcinoma; ICGR 15, indocyanine green retention at $15 \mathrm{~min}$; AST, aspartate aminotransferase; ALT, alanine aminotransferase; AFP, $\alpha$-fetoprotein; DCP, des- $\gamma$-carboxy prothrombin. ${ }^{a}$ log-rank test.

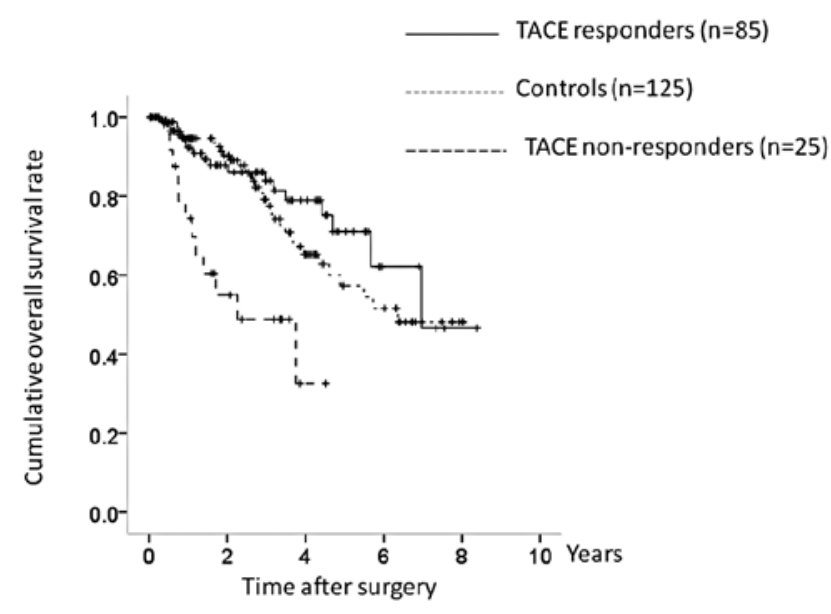

Figure 4. Overall survival (OS) in the transcatheter arterial chemoembolization (TACE) responder group $(n=85)$, the TACE non-responder group $(n=25)$ and the control group. There were significant differences between the three groups in terms of OS (TACE responders vs. controls, $\mathrm{P}=0.381$; controls vs. TACE non-responders, $\mathrm{P}<0.001$; TACE responders vs. TACE non-responders, $\mathrm{P}<0.001$; overall significance, $\mathrm{P}<0.001$; log rank test).

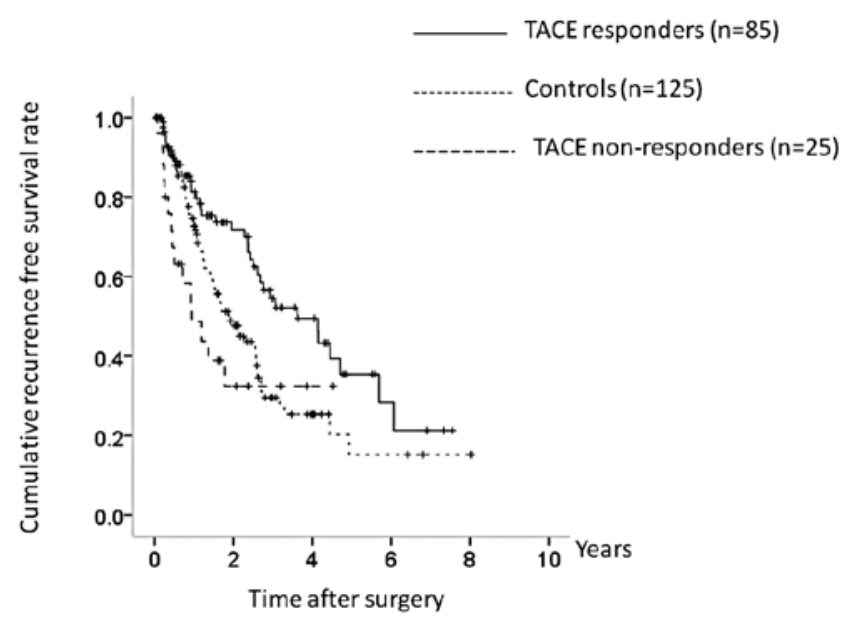

Figure 5. Recurrence-free survival (RFS) in the transcatheter arterial chemoembolization (TACE) responder group $(n=85)$, the TACE non-responder group $(n=25)$ and the control group. There were significant differences between the three groups in terms of RFS (TACE responders vs. controls, $\mathrm{P}=0.006$; controls vs. TACE non-responders, $\mathrm{P}=0.190$; TACE responders vs. TACE non-responders, $\mathrm{P}=0.004$; overall significance, $\mathrm{P}=0.004$ ). 
Table IV. Multivariate analyses contributing to OS after surgical resection.

\begin{tabular}{|c|c|c|c|}
\hline Variables & $\begin{array}{c}\text { Hazard } \\
\text { ratio }\end{array}$ & $95 \% \mathrm{CI}$ & P-value \\
\hline
\end{tabular}

Pretreatment therapy

$\begin{array}{llll}\text { TACE responders } & 2.433 & 1.161-5.102 & 0.018 \\ \text { TACE non-responders } & 0.374 & 0.164-0.851 & 0.019 \\ \text { Controls } & 1.000 & & \end{array}$

HCC stage

Stage I, II

Stage III, IV

1.000

Maximum tumor size $(\mathrm{cm})$

$\geq 4$

$<4$

0.766
1.000

$0.252-3.302 \quad 0.889$

$0.416-1.411 \quad 0.393$

Tumor number

Single

Multiple

1.000

0.656

$0.185-2.330 \quad 0.514$

Total bilirubin $(\mathrm{mg} / \mathrm{dl})$

$\geq 1.0$

$<1.0$

0.413

1.000

$0.231-0.740 \quad 0.003$

Serum albumin $(\mathrm{g} / \mathrm{dl})$

$\geq 4.0$

$<4.0$

2.579

1.000

$1.446-4.599 \quad 0.001$

AFP (ng/ml)

$\geq 100$

$<100$

0.486

1.000

$\mathrm{DCP}(\mathrm{mAU} / \mathrm{ml})$

$\geq 100$

$<100$

0.627

1.000

$0.320-1.229 \quad 0.174$

$0.280-0.846 \quad 0.011$

(174)

Microscopic vascular

invasion

$\begin{array}{llll}\text { Yes } & 0.491 & 0.269-0.899 & 0.021 \\ \text { No } & 1.000 & & \end{array}$

TACE, transcatheter arterial chemoembolization; HCC, hepatocellular carcinoma; AFP, $\alpha$-fetoprotein; DCP, des- $\gamma$-carboxy prothrombin; 95\% CI, 95\% confidence interval. a Cox proportional hazards model.

number $(\mathrm{P}<0.001)$, total bilirubin $\geq 1 \mathrm{mg} / \mathrm{dl}(\mathrm{P}=0.041)$, aspartate aminotransferase $\geq 50 \mathrm{IU} / 1(\mathrm{P}=0.005)$, alanine aminotransferase $\geq 50 \mathrm{IU} / 1(\mathrm{P}=0.012), \mathrm{AFP} \geq 100 \mathrm{ng} / \mathrm{ml}(\mathrm{P}=0.036)$ and microscopic vascular invasion $(\mathrm{P}=0.021)$ as significant factors contributing to RFS after SR (Table III). Multivariate analysis of the nine factors found to be significant in univariate analysis confirmed pretreatment therapy (TACE non-responder, $\mathrm{P}=0.039)$, tumor number $(\mathrm{P}=0.038)$ and $\mathrm{AFP} \geq 100 \mathrm{ng} / \mathrm{ml}$ $(\mathrm{P}=0.043)$ as significant contributors to RFS. The HRs and 95\% CIs for these factors are detailed in Table V.

Comparison of baseline characteristics between TACE responders and non-responders. The baseline characteristics of the TACE responders $(n=85)$ and non-responders $(n=25)$ are shown in Table VI. There were significant differences between
Table V. Multivariate analyses contributing to RFS after surgical resection.

\begin{tabular}{|c|c|c|c|}
\hline Variables & $\begin{array}{l}\text { Hazard } \\
\text { ratio }\end{array}$ & $95 \% \mathrm{CI}$ & P-value \\
\hline \multicolumn{4}{|l|}{ Pretreatment therapy } \\
\hline TACE responders & 1.075 & 0.593-1.949 & 0.811 \\
\hline TACE non-responders & 0.508 & $0.267-0.966$ & 0.039 \\
\hline Controls & 1.000 & & \\
\hline \multicolumn{4}{|l|}{ HCC stage } \\
\hline Stage I, II & 1.000 & & \\
\hline Stage III, IV & 0.845 & $0.319-2.238$ & 0.735 \\
\hline \multicolumn{4}{|l|}{ Maximum tumor size (cm) } \\
\hline$\geq 4$ & 0.872 & $0.584-1.302$ & 0.503 \\
\hline$<4$ & 1.000 & & \\
\hline \multicolumn{4}{|l|}{ Tumor number } \\
\hline Single & 1.000 & & \\
\hline Multiple & 0.513 & $0.094-0.953$ & 0.038 \\
\hline \multicolumn{4}{|l|}{ Total bilirubin (mg/dl) } \\
\hline$\geq 1.0$ & 0.801 & $0.516-1.246$ & 0.325 \\
\hline$<1.0$ & 1.000 & & \\
\hline \multicolumn{4}{|l|}{ AST (IU/1) } \\
\hline$\geq 50$ & 0.833 & $0.486-1.429$ & 0.508 \\
\hline$<50$ & 1.000 & & \\
\hline \multicolumn{4}{|l|}{ ALT (IU/l) } \\
\hline$\geq 50$ & 0.751 & $0.434-1.300$ & 0.307 \\
\hline$<50$ & 1.000 & & \\
\hline \multicolumn{4}{|l|}{$\mathrm{AFP}(\mathrm{ng} / \mathrm{ml})$} \\
\hline$\geq 100$ & 0.616 & $0.379-0.970$ & 0.043 \\
\hline$<100$ & 1.000 & & \\
\hline \multicolumn{4}{|l|}{$\begin{array}{l}\text { Microscopic vascular } \\
\text { invasion }\end{array}$} \\
\hline Yes & 0.857 & $0.568-1.292$ & 0.462 \\
\hline No & 1.000 & & \\
\hline
\end{tabular}

TACE, transcatheter arterial chemoembolization; AST, aspartate aminotransferase; ALT, alanine aminotransferase; AFP, $\alpha$-fetoprotein; 95\% CI, 95\% confidence interval. ${ }^{\mathrm{a}} \mathrm{Cox}$ proportional hazards model.

the groups in terms of HCC stage, maximum tumor size, pretreatment AFP value and pretreatment DCP value, indicating that TACE non-responders had more advanced tumor characteristics than TACE responders.

Causes of death. Twenty-nine patients in the TACE group (26.4\%) died during the follow-up period. The causes of death were $\mathrm{HCC}$ recurrence in 21 patients, liver failure in 6 patients and other causes in 2 patients. Thirty-two patients in the control group (25.6\%) died during the follow-up period, and the causes of death were $\mathrm{HCC}$ recurrence in 21 patients, liver failure in 7 patients and other causes in 4 patients.

HCC recurrence in the TACE and control groups. Fiftythree patients in the TACE group (48.2\%) and $69(55.2 \%)$ in 
Table VI. Baseline characteristics between the TACE responder group and the TACE non-responder group.

\begin{tabular}{lccc}
\hline & TACE responders $(\mathrm{n}=85)$ & TACE non-responders $(\mathrm{n}=25)$ & P-value \\
\hline Gender (male/female) & $66 / 19$ & $20 / 5$ & $1.000^{\mathrm{a}}$ \\
Age (years) & $67.2 \pm 10.7$ & $69.1 \pm 8.0$ & $0.452^{\mathrm{b}}$ \\
HCC stage (I/II/III/IV) & $5 / 47 / 29 / 4$ & $0 / 10 / 9 / 6$ & $0.019^{\mathrm{a}}$ \\
Etiology (HBV/HCV/non-Bnon-C) & $17 / 50 / 18$ & $2 / 14 / 9$ & $0.178^{\mathrm{a}}$ \\
Child-Pugh classification (A/B) & $84 / 1$ & $24 / 1$ & $0.405^{\mathrm{a}}$ \\
Tumor number (single/multiple) & $54 / 31$ & $11 / 14$ & $0.106^{\mathrm{a}}$ \\
Maximum tumor size (cm) & $4.1 \pm 1.9$ & $8.1 \pm 4.5$ & $<0.001^{\mathrm{b}}$ \\
AST (IU/l) & $60.5 \pm 40.9$ & $56.0 \pm 38.4$ & $0.627^{\mathrm{b}}$ \\
ALT (IU/l) & $55.5 \pm 48.5$ & $47.6 \pm 31.0$ & $0.446^{\mathrm{b}}$ \\
Serum albumin (g/dl) & $3.97 \pm 0.49$ & $3.82 \pm 0.61$ & $0.183^{\mathrm{b}}$ \\
Total bilirubin (mg/dl) & $0.78 \pm 0.37$ & $0.80 \pm 0.45$ & $0.781^{\mathrm{b}}$ \\
Prothrombin time (\%) & $89.0 \pm 14.1$ & $90.3 \pm 12.7$ & $0.667^{\mathrm{b}}$ \\
Platelets (x10 $\left./ \mathrm{mm}^{3}\right)$ & $15.0 \pm 8.0$ & $15.0 \pm 7.2$ & $0.983^{\mathrm{b}}$ \\
ICGR 15 (\%) & $12.8 \pm 8.5$ & $12.5 \pm 11.9$ & $0.897^{\mathrm{b}}$ \\
AFP (ng/ml) & $1,114.0 \pm 3,917.9$ & $8,712.7 \pm 31,280.1$ & $0.030^{\mathrm{b}}$ \\
DCP (mAU/ml) & $3,197.0 \pm 11,343.4$ & $19,495.1 \pm 41,923.6$ & $0.002^{\mathrm{b}}$ \\
\hline
\end{tabular}

TACE, transcatheter arterial chemoembolization; HCC, hepatocellular carcinoma; HBV, hepatitis B virus; HCV, hepatitis C virus; AST, aspartate aminotransferase; ALT, alanine aminotransferase; ICGR 15, indocyanine green retention at 15 min; AFP, $\alpha$-fetoprotein; DCP, des- $\gamma$ -

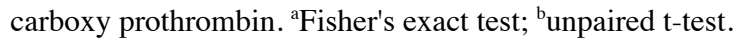

the control group had HCC recurrence during the follow-up period. The patterns of HCC recurrence after SR in the TACE group were as follows: single HCC recurrence in the liver in 12 patients; multiple HCC recurrences in the liver in 29 patients; multiple HCC recurrences in the liver with lung metastases in 7 patients; multiple $\mathrm{HCC}$ recurrences in the liver with brain metastases in 1 patient; and multiple $\mathrm{HCC}$ recurrences in the liver with multiple lymph node (LN) metastases in 4 patients. The patterns of HCC recurrence after SR in the control group were: single HCC recurrence in the liver in 32 patients; single HCC recurrence with invasion of the right hepatic vein in 1 patient; multiple $\mathrm{HCC}$ recurrences in the liver in 33 patients; multiple $\mathrm{HCC}$ recurrences in the liver with $\mathrm{LN}$ metastases in 1 patient; multiple HCC recurrences in the liver with portal vein invasion in 1 patient; and multiple HCC recurrences in the liver with lung metastases in 1 patient.

The treatment methods for the first HCC recurrence in the TACE group were SR in 2 patients, radiofrequency ablation (RFA) in 23 patients, TACE in 16 patients, percutaneous ethanol injection (PEI) in 2 patients, systemic chemotherapy in 7 patients and no specific treatment in 3 patients. The treatment methods used in the control group were SR in 6 patients, RFA in 36 patients, TACE in 15 patients, PEI in 2 patients, systemic chemotherapy in 4 patients and no specific treatment in 6 patients.

Operation time, surgical blood loss and hospitalization period in the TACE and control groups. The mean operation times were $259.5 \pm 74.5 \mathrm{~min}$ in the TACE group and $269.1 \pm 87.8 \mathrm{~min}$ in the control group $(\mathrm{P}=0.881)$. The mean surgical blood loss was $764.1 \pm 713.5 \mathrm{ml}$ in the TACE group and $874.2 \pm 886.8 \mathrm{ml}$ in the control group $(\mathrm{P}=0.334)$. The mean periods from surgery until discharge were $17.9 \pm 15.8$ days in the TACE group and $16.6 \pm 10.4$ days in the control group $(\mathrm{P}=0.447)$ (Table II).

TACE and surgery-related serious adverse events (SAES). The interval from TACE until surgery in the TACE group was $38.0 \pm 17.6$ days, and no patient was prevented from undergoing $\mathrm{SR}$ as a result of TACE-related complications. Surgeryrelated SAEs in the TACE group included abscess formation in 5 patients, bile leakage in 4 patients, refractory ascites in 2 patients, aspiration pneumonia in 2 patients, gastrointestinal bleeding in 2 patients, acute heart failure in 1 patient and perforation of the small intestine in 1 patient. Equivalent complications in the control group included abscess formation in 3 patients, bile leakage in 4 patients, refractory ascites in 6 patients, aspiration pneumonia in 2 patients, acute respiratory distress syndrome in 1 patient and brain infarction in 1 patient. All these SAEs improved during the same hospitalization. There was no significant difference between the groups in terms of SAEs related to surgery $(\mathrm{P}=0.714)$.

Subgroup analyses of $O S$ and RFS in patients with HCC stage I or II. TACE non-responders had more advanced tumor characteristics than TACE responders, and we therefore performed subgroup analyses in patients with HCC stage I or II $(n=155)$ and HCC stage III or IV $(n=80)$. Patients with HCC stage I or II comprised 52 TACE responders, 10 TACE non-responders and 93 controls. Although the TACE nonresponders had a poorer prognosis in terms of OS, there was no overall significant difference between the three groups (TACE responders vs. controls, $\mathrm{P}=0.523$; controls vs. TACE 


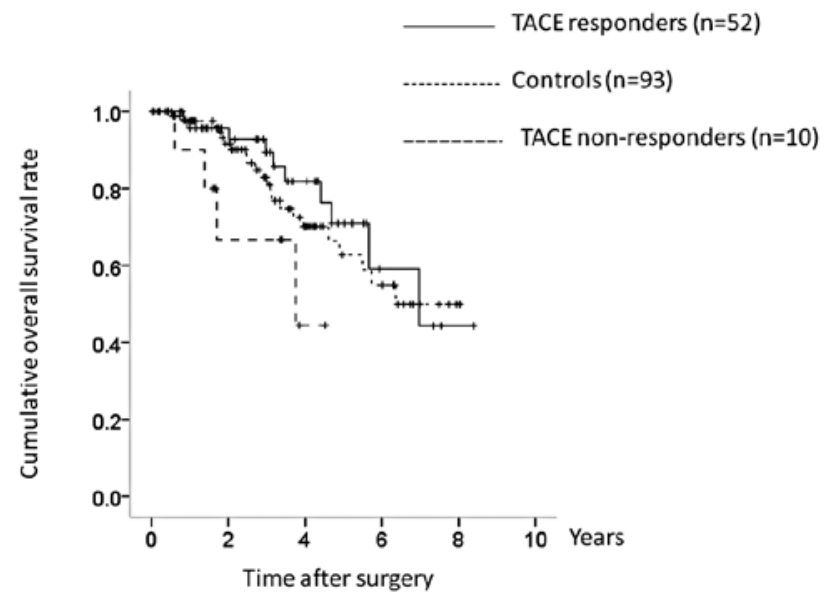

Figure 6. Subgroup analyses in patients with HCC stage I or II in terms of overall survival (OS). Patients with HCC stage I or II ( $n=155)$ included 52 TACE responders, 10 TACE non-responders and 93 controls. Although the TACE non-responder patients had a poorer prognosis in terms of OS, there were no significant differences between the three groups (TACE responders vs. controls, $\mathrm{P}=0.523$; controls vs. TACE non-responders, $\mathrm{P}=0.118$; TACE responders vs. TACE non-responders, $\mathrm{P}=0.040$; overall significance, $\mathrm{P}=0.148$; long rank test).

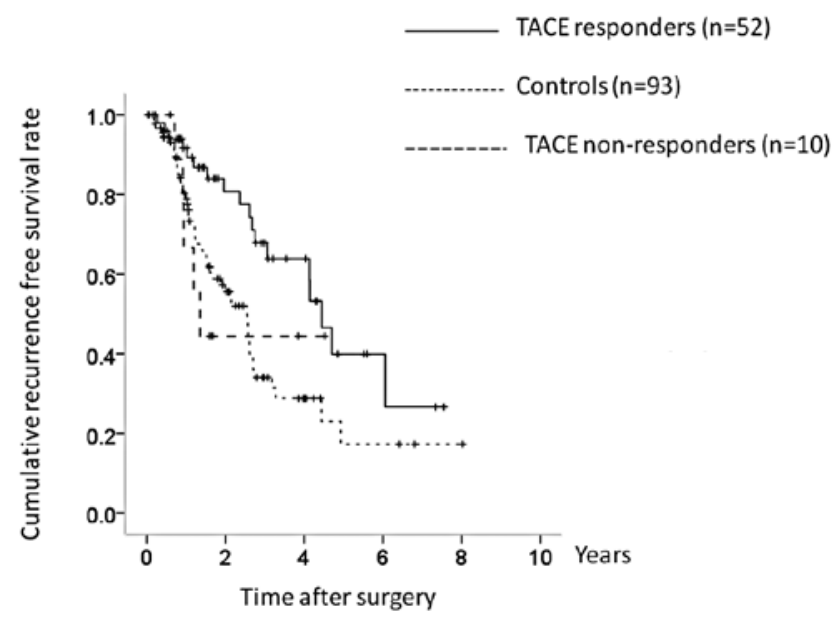

Figure 7. Subgroup analyses in patients with HCC stage I or II in terms of recurrence-free survival (RFS). There were significant differences between these three groups in terms of RFS (TACE responders vs. controls, $\mathrm{P}=0.003$; controls vs. TACE non-responders, $\mathrm{P}=0.992$; TACE responders vs. TACE non-responders, $\mathrm{P}=0.105$; overall significance, $\mathrm{P}=0.013$; long rank test).

non-responders, $\mathrm{P}=0.118$; TACE responders vs. TACE nonresponders, $\mathrm{P}=0.040$; overall significance, $\mathrm{P}=0.148$ ) (Fig. 6). However, there were significant differences between the three groups in terms of RFS (TACE responders vs. controls, $\mathrm{P}=0.003$; controls vs. TACE non-responders, $\mathrm{P}=0.992$; TACE responders vs. TACE non-responders, $\mathrm{P}=0.105$; overall significance, $\mathrm{P}=0.013$ ) (Fig. 7).

Subgroup analyses of OS and RFS in patients with HCC stage III or IV. The patients with HCC stage III or IV $(\mathrm{n}=80)$ included 33 TACE responders, 15 TACE non-responders and 32 controls. There were significant differences in OS between the three groups (TACE responders vs. controls,

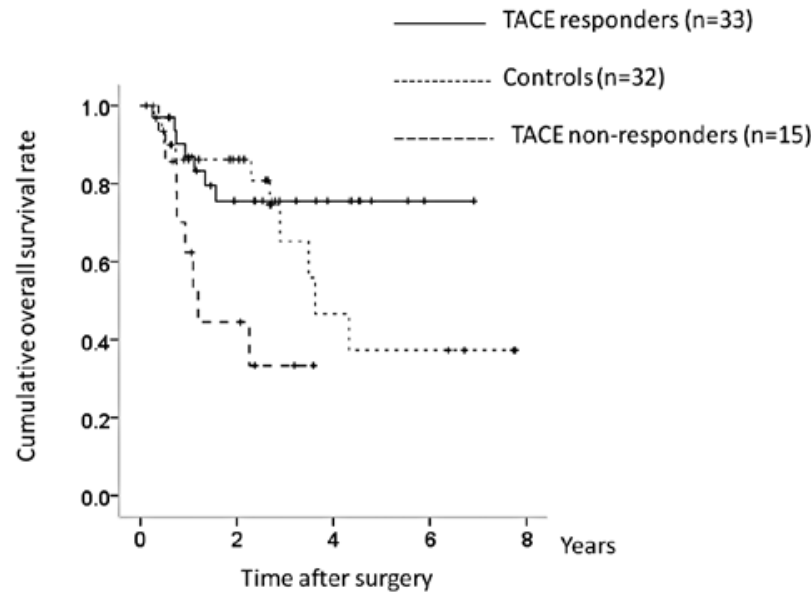

Figure 8. Subgroup analyses in patients with HCC stage III or IV in terms of overall survival (OS). Patients with HCC stage III or IV $(n=80)$ included 33 TACE responders, 15 TACE non-responders and 32 controls. There were significant differences between these three groups in terms of OS (TACE responders vs. controls, $\mathrm{P}=0.267$; controls vs. TACE non-responders, $\mathrm{P}=0.025$ TACE responders vs. TACE non-responders, $\mathrm{P}=0.010$; overall significance, $\mathrm{P}=0.011$; long rank test).

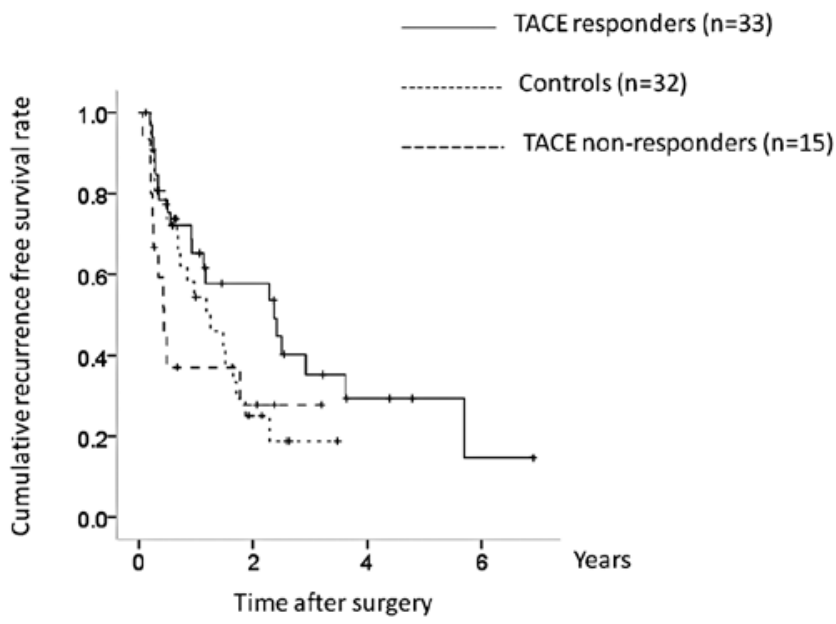

Figure 9. Subgroup analyses in patients with HCC stage III or IV in terms of recurrence-free survival (RFS). Although the TACE non-responders had a poorer prognosis in terms of RFS, the difference was not significant (TACE responders vs. controls, $\mathrm{P}=0.106$; controls vs. TACE non-responders, $\mathrm{P}=0.462$; TACE responders vs. TACE non-responders, $\mathrm{P}=0.060$; overall significance, $\mathrm{P}=0.116$; long rank test).

$\mathrm{P}=0.267$; controls vs. TACE non-responders, $\mathrm{P}=0.025$; TACE responders vs. TACE non-responders, $\mathrm{P}=0.010$; overall significance, $\mathrm{P}=0.011$ ) (Fig. 8). In terms of RFS, although TACE non-responders had a poorer prognosis, the difference was not significant (TACE responders vs. controls, $\mathrm{P}=0.106$; controls vs. TACE non-responders, $\mathrm{P}=0.462$; TACE responders vs. TACE non-responders, $\mathrm{P}=0.060$; overall significance, $\mathrm{P}=0.116$ ) (Fig. 9).

\section{Discussion}

To the best of our knowledge, the present study represents one of the largest comparative studies on the influence of preop- 
erative TACE on the survival of patients with resectable HCC $(3,10,12,13,19-21)$. Although four randomized controlled trials have investigated the effects of pretreatment TACE on survival after SR $(3,10,12,13)$ and similarly concluded that pretreatment with TACE did not improve survival after SR, the sample sizes, TACE procedures and baseline clinical characteristics differed among these studies. Hence, the efficacy of pretreatment TACE on survival after SR remains unclear.

In this study, multivariate analysis identified TACE responder and TACE non-responder in terms of OS, and TACE non-responder in terms of RFS, as significant independent prognostic factors after SR. Moreover, in terms of RFS in stage I or II HCC patients and OS in stage III or IV HCC patients, the overall differences reached significance in univariate analysis. These results suggest that the therapeutic efficacy of pretreatment TACE is associated with clinical outcome after SR.

The extent of tumor vascularization is significantly associated with the degree of TACE efficacy, and a high degree of vascularization is thus considered to be a predictive sign for response to TACE (22). Preoperative TACE may thus be recommended in HCC patients with a high degree of tumor vascularity (23), although Adachi et al reported that preoperative TACE should be avoided as incomplete tumor necrosis promotes the hematogenous spread of residual tumor cells during SR (24).

Several studies have reported that serum vascular endothelial growth factor (VEGF) can act as a prognostic factor for the treatment of HCC (25-27). Sergio et al (22) also demonstrated that when TACE for HCC was ineffective, it might induce a significant increase in serum VEGF levels and affect patient survival. TACE non-responders in the present study may thus have had a poor prognosis associated with increased serum VEGF levels. It has also been suggested that preoperative TACE should not be performed in patients with a low degree of vascularization. Further studies are needed to clarify this issue.

In terms of tumor histology, the current study included 13 patients $(52.0 \%)$ with poorly differentiated HCC in the TACE non-responder group and 22 patients (25.9\%) with poorly differentiated HCC in the TACE responder group $(\mathrm{P}=0.017)$. Patients with poorly differentiated HCC would be expected to have a poorer prognosis because of their poor response to TACE.

Liver function parameters reflected by serum bilirubin and serum albumin were significant independent factors linked to OS in the present study. Several studies have investigated the importance of maintaining liver function on survival after surgery for HCC $(28,29)$. Our results were consistent with previous reports; in HCC patients with poor liver function, branched chain amino acid therapy to maintain liver function may be effective to optimize the clinical outcomes (7).

High pretreatment AFP level was an independent prognostic factor in terms of both OS and RFS in our study. HCC patients with high AFP levels had poorer tumor histology and larger tumor mass (30), which may be associated with their poorer clinical outcome. Microvascular invasion was a significant prognostic factor in univariate and multivariate analyses in terms of OS, and in the univariate analysis in terms of RFS. Lim et al reported that microvascular invasion was an adverse predictor of OS and RFS following SR for HCC (31). Our results were in agreement with their reports. Careful monitoring for $\mathrm{HCC}$ recurrence will thus be needed in patients with microvascular invasion, and patients with high pretreatment AFP levels.

Zhou et al reported that several patients in their randomized controlled trial could not undergo definitive surgery because of tumor progression after TACE or because of TACE-related SAEs. However, no patients in the current study were unable to undergo surgery (3). Moreover, there were no significant differences between the TACE and control groups in terms of operation time, blood loss during surgery and hospitalization period. Our study results, therefore, suggest that the TACE procedure was safe in patients with resectable HCC.

The present study has several limitations. First, it is a retrospective study. Second, the follow-up period was relatively short for survival analysis. Third, the sample sizes of the TACE responder, TACE non-responder, and control groups were not balanced. However, despite these limitations, the results of this study demonstrated that the therapeutic efficacy of preoperative TACE may be associated with clinical outcome after SR in patients with HCC. Preoperative TACE may thus be a prognostic factor in patients with resectable HCC after SR.

\section{Acknowledgements}

The authors would like to thank all the staff in the angiography room and the operation room of the Osaka Red Cross Hospital for their valuable support.

\section{References}

1. Livraghi T, Mäkisalo H and Line PD: Treatment options in hepatocellular carcinoma today. Scand J Surg 100: 22-29, 2011.

2. El-Serag HB: Epidemiology of viral hepatitis and hepatocellular carcinoma. Gastroenterology 142: 1264-1273, 2012.

3. Zhou WP, Lai EC, Li AJ, Fu SY, Zhou JP, Pan ZY, Lau WY and Wu MC: A prospective, randomized, controlled trial of preoperative transarterial chemoembolization for resectable large hepatocellular carcinoma. Ann Surg 249: 195-202, 2009.

4. Nishikawa H, Osaki Y, Kita R, Kimura T, Inuzuka T, Takeda H, Nakajima J, Matsuda F, Sakamoto A, Henmi S, Hatamaru K, Saito $S$ and Nasu A: Transcatheter arterial infusion chemotherapy prior to radiofrequency thermal ablation for single hepatocellular carcinoma reduces the risk of intrahepatic distant recurrence. Int J Oncol 41: 903-909, 2012.

5. de Lope CR, Tremosini S, Forner A, Reig M and Bruix J: Management of HCC. J Hepatol 56 (Suppl 1): S75-S87, 2012.

6. Takayasu K, Arii S, Ikai I, Omata M, Okita K, Ichida T, Matsuyama Y, Nakanuma Y, Kojiro M and Makuuchi M: Prospective cohort study of transarterial chemoembolization for unresectable hepatocellular carcinoma in 8510 patients. Gastroenterology 131: 461-469, 2006.

7. Nishikawa H, Osaki Y, Inuzuka T, Takeda H, Nakajima J, Matsuda F, Henmi S, Sakamoto A, Ishikawa T, Saito S, Kita R and Kimura T: Branched-chain amino acid treatment before transcatheter arterial chemoembolization for hepatocellular carcinoma. World J Gastroenterol 18: 1379-1384, 2012.

8. Llovet JM and Bruix J: Systematic review of randomized trials for unresectable hepatocellular carcinoma: chemoembolization improves survival. Hepatology 37: 429-442, 2003.

9. Bruix J and Sherman M; Practice Guidelines Committee, American Association for the Study of Liver Diseases: Management of hepatocellular carcinoma. Hepatology 42: 1208-1236, 2005.

10. Kaibori M, Tanigawa N, Kariya S, Ikeda H, Nakahashi Y, Hirohara J, Koreeda C, Seki T, Sawada S, Okazaki K and Kwon AH: A prospective randomized controlled trial of preoperative wholeliver chemolipiodolization for hepatocellular carcinoma. Dig Dis Sci 57: 1404-1412, 2012. 
11. Yamanaka N, Okamoto E, Fujihara S, Kato T, Fujimoto J, Oriyama T, Mitsunobu M, Toyosaka A, Uematsu K and Yamamoto K: Do the tumor cells of hepatocellular carcinomas dislodge into the portal venous stream during hepatic resection? Cancer 70: 2263-2267, 1992.

12. Yamasaki S, Hasegawa H, Kinoshita H, Furukawa M, Imaoka S, Takasaki K, Kakumoto Y, Saitsu H, Yamada R, Oosaki Y, Arii S, Okamoto E, Monden M, Ryu M, Kusano S, Kanematsu T, Ikeda K, Yamamoto M, Saoshiro T and Tsuzuki T: A prospective randomized trial of the preventive effect of pre-operative transcatheter arterial embolization against recurrence of hepatocellular carcinoma. Jpn J Cancer Res 87: 206-211, 1996.

13. Wu CC, Ho YZ, Ho WL, Wu TC, Liu TJ and P'eng FK: Preoperative transcatheter arterial chemoembolization for resectable large hepatocellular carcinoma: a reappraisal. Br J Surg 82: 122-126, 1995.

14. Yamasaki T, Kurokawa F, Shirahashi H, Kusano N, Hironaka K and Okita K: Percutaneous radiofrequency ablation therapy with combined angiography and computed tomography assistance for patients with hepatocellular carcinoma. Cancer 91: 1342-1348, 2001.

15. Liver Cancer Study Group of Japan: The general rules for the clinical and pathological study of primary liver cancer. Jpn J Surg 19: 98-129, 1989.

16. Kudo M and Okanoue T: Management of hepatocellular carcinoma in Japan: consensus-based clinical practice manual proposed by the Japan Society of Hepatology. Oncology 72 (Suppl 1): 2-15, 2007.

17. Kudo M, Kubo S, Takayasu K, Sakamoto M, Tanaka M, Ikai I, Furuse J, Nakamura K and Makuuchi M; Liver Cancer Study Group of Japan (Committee for Response Evaluation Criteria in Cancer of the Liver, Liver Cancer Study Group of Japan): Response Evaluation Criteria in Cancer of the Liver (RECICL) proposed by the Liver Cancer Study Group of Japan (2009 Revised Version). Hepatol Res 40: 686-692, 2010.

18. Hu BS, Chen K, Tan HM, Ding XM and Tan JW: Comparison of laparoscopic vs open liver lobectomy (segmentectomy) for hepatocellular carcinoma. World J Gastroenterol 17: 4725-4728, 2011.

19. Sasaki A, Iwashita Y, Shibata K, Ohta M, Kitano S and Mori M: Preoperative transcatheter arterial chemoembolization reduces long-term survival rate after hepatic resection for resectable hepatocellular carcinoma. Eur J Surg Oncol 32: 773-779, 2006.

20. Choi GH, Kim DH, Kang CM, Kim KS, Choi JS, Lee WJ and Kim BR: Is preoperative transarterial chemoembolization needed for a resectable hepatocellular carcinoma? World J Surg 31: 2370-2377, 2007.

21. Sugo H, Futagawa S, Beppu T, Fukasawa M and Kojima K: Role of preoperative transcatheter arterial chemoembolization for resectable hepatocellular carcinoma: relation between postoperative course and the pattern of tumor recurrence. World J Surg 27: 1295-1299, 2003.
22. Sergio A, Cristofori C, Cardin R, Pivetta G, Ragazzi R, Baldan A, Girardi L, Cillo U, Burra P, Giacomin A and Farinati F: Transcatheter arterial chemoembolization (TACE) in hepatocellular carcinoma (HCC): the role of angiogenesis and invasiveness. Am J Gastroenterol 103: 914-921, 2008.

23. Zhang Z, Liu Q, He J, Yang J, Yang G and Wu M: The effect of preoperative transcatheter hepatic arterial chemoembolization on disease-free survival after hepatectomy for hepatocellular carcinoma. Cancer 89: 2606-2612, 2000.

24. Adachi E, Matsumata T, Nishizaki T, Hashimoto H, Tsuneyoshi M and Sugimachi K: Effects of preoperative transcatheter hepatic arterial chemoembolization for hepatocellular carcinoma. The relationship between postoperative course and tumor necrosis. Cancer 72: 3593-3598, 1998.

25. Chao Y, Li CP, Chau GY, Chen CP, King KL, Lui WY, Yen SH, Chang FY, Chan WK and Lee SD: Prognostic significance of vascular endothelial growth factor, basic fibroblast growth factor, and angiogenin in patients with resectable hepatocellular carcinoma after surgery. Ann Surg Oncol 10: 355-362, 2003.

26. Poon RT, Lau C, Yu WC, Fan ST and Wong J: High serum levels of vascular endothelial growth factor predict poor response to transarterial chemoembolization in hepatocellular carcinoma: a prospective study. Oncol Rep 11: 1077-1084, 2004.

27. Shim JH, Park JW, Kim JH, An M, Kong SY, Nam BH, Choi JI, Kim HB, Lee WJ and Kim CM: Association between increment of serum VEGF level and prognosis after transcatheter arterial chemoembolization in hepatocellular carcinoma patients. Cancer Sci 99: 2037-2044, 2008.

28. Ikai I, Arii S, Kojiro M, Ichida T, Makuuchi M, Matsuyama Y, Nakanuma Y, Okita K, Omata M, Takayasu K and Yamaoka Y: Reevaluation of prognostic factors for survival after liver resection in patients with hepatocellular carcinoma in a Japanese nationwide survey. Cancer 101: 796-802, 2004

29. Gluer AM, Cocco N, Laurence JM, Johnston ES, Hollands MJ, Pleass HC, Richardson AJ and Lam VW: Systematic review of actual 10-year survival following resection for hepatocellular carcinoma. HPB 14: 285-290, 2012.

30. Zhou L, Liu J and Luo F: Serum tumor markers for detection of hepatocellular carcinoma. World J Gastroenterol 12: 1175-1181, 2006.

31. Lim KC, Chow PK, Allen JC, Chia GS, Lim M, Cheow PC, Chung AY, Ooi LL and Tan SB: Microvascular invasion is a better predictor of tumor recurrence and overall survival following surgical resection for hepatocellular carcinoma compared to the Milan criteria. Ann Surg 254: 108-113, 2011. 